\title{
The velocity field of baryonic gas in the universe
}

\author{
Bryan Kim ${ }^{1}$, Ping $\mathrm{He}^{2,3}$, Jesús Pando ${ }^{1}$, Long-Long Feng ${ }^{2,4}$ and Li-Zhi Fang ${ }^{3}$
}

\begin{abstract}
The dynamic evolution of the baryonic intergalactic medium (IGM) caused by the underlying dark matter gravity is governed by the Navier-Stokes equations in which many cooling and heating processes are involved. However, it has long been recognized that the growth mode dynamics of cosmic matter clustering can be sketched by a random force driven Burgers' equation if cooling and heating are ignored. Just how well the dynamics of the IGM can be described as a Burgers fluid has not been fully investigated probably because cooling and heating are essential for a detailed understanding of the IGM. Using IGM samples produced by a cosmological hydrodynamic simulation in which heating and cooling processes are properly accounted for, we show that the IGM velocity field in the nonlinear regime shows the features of a Burgers fluid, that is, when the Reynolds number is high, the velocity field consists of an ensemble of shocks. Consequently, (1) the IGM velocity $v$ is generally smaller than that of dark matter; (2) for the smoothed field, the IGM velocity shows tight correlation with dark matter given by $v \simeq s v_{d m}$, with $s<1$, such that the lower the redshift, the smaller $s$; (3) the velocity PDFs are asymmetric between acceleration and deceleration events; (4) the PDF of velocity difference $\Delta v=$ $v(x+r)-v(x)$ satisfies the scaling relation for a Burgers fluid, i.e., $P(\Delta v)=\left(1 / r^{y}\right) F\left(\Delta v / r^{y}\right)$. We find the scaling function and parameters for the IGM which are applicable to the entire scale range of the samples $\left(0.26-8 \mathrm{~h}^{-1} \mathrm{Mpc}\right)$. These properties show that the similarity mapping between the IGM and dark matter is violated on scales much larger than the Jeans length of the IGM.
\end{abstract}

Subject headings: cosmology: theory - large-scale structure of universe

\section{Introduction}

The evolution of large scale structure in the universe is largely governed by the gravitational clustering of dark matter. The collapsing of the dark matter halos form the potential wells that trap baryonic gas, or the intergalactic medium (IGM), and form light-emitting objects. Clearly understanding the dynamics of the IGM embedded in the underlying dark matter field is essential in

\footnotetext{
${ }^{1}$ Department of Physics, DePaul University, Chicago Il, 60614

${ }^{2}$ National Astronomical Observatories, Chinese Academy of Science, Chao-Yang District, Beijing, 100012, P.R. China

${ }^{3}$ Department of Physics, University of Arizona, Tucson, AZ 85721

${ }^{4}$ Purple Mountain Observatory, Chinese Academy of Sciences, Nanjing, 210008
}

large scale structure study.

The cosmic baryonic gas is a Navier-Stokes fluid. Unlike an ordinary fluid, the dynamic behavior of the IGM is dominated by the growth modes of dark matter. It has long been recognized that the growth mode dynamics of cosmic baryonic gas can be approximately described by the random-force-driven Burgers' equation. Zeldovich's pancake theory and the adhesion model first indicated that the non-linear evolution of the gravitational clustering of cosmic matter could be sketched by a Burgers' equation (Gurbatov, Saichev, \& Shandarin 1989; Vergassola et al. 1994). By considering cosmic matter as dissipative, Berera \& Fang (1994) showed that the dynamical equations of the velocity fields of cosmic matter are essentially a variant of the randomforce-driven Burgers equation or the KPZ equation (Kardar et al 1986). Later, with a two- 
component (dark matter and IGM) generalization of the adhesion model, it was found that the velocity potential of the baryonic matter is described by the Burgers equation driven by the gravitational potential of dark matter (Jones, 1999; Matarrese and Mohayaee 2002).

Observations also reveal that the IGM shows features that hint at a field governed by the dynamics of Burgers' equation. For example, the transmitted flux of QSO's Ly $\alpha$ absorption is found to be significantly intermittent and its probability distribution functions (PDF) are long tailed and probably lognormal (Jamkhedkar et al. 2000, Pando et al. 2002, Feng et al. 2003, Jamkhedkar et al. 2003). This is consistent with the intermittent spatial and temporal behavior of a developed Burgers fluid (Polyakov 1995, Bouchaud, Mézard \& Parisi 1995, Gurarie \& Migdal 1996, Balkovsky et al. 1997, Yakhot 1998, Frisch, Bec \& Villone 1999). Moreover, if the random-force in the Burgers' equation for the baryonic matter arises from the gravity of dark matter, the statistical properties of the baryonic matter in the non-linear regime will decouple from those of the underlying dark matter. This mechanism leads to a breaking of similarity between the IGM and dark matter on scales larger than the Jeans length (He et al. 2005). This large scale dis-similarity has also been found from observations of the entropy floor (Ponman, et al. 1999), X-ray luminosity-temperature relation of galactic clusters; the baryon fraction of clusters (Ettori \& Fabian 1999, Sand et al. 2003); and the multi-phase IGM temperature field (Zhan et al. 2003) etc.

However, there is a difference between the theoretical models and the observations. In all the above-mentioned theoretical approaches, heating and cooling processes of the IGM are ignored. That is, the dynamical equation of the growth modes of the IGM without heating and cooling can be approximated by a Burgers' equation. However, heating and cooling are essential for understanding all details of the IGM. Therefore, an important question is how well the dynamics of IGM can be described as a Burgers fluid that has undergone significant heating and cooling evolution. To investigate this question, we focus on the velocity field or velocity potential because predictions based on Burgers dynamics directly concern the velocity and velocity potential. We use samples of the IGM velocity field produced by cosmological hydrodynamical simulations in which all heating and cooling processes are properly accounted for and investigate whether the IGM displays the common features of a Burgers' fluid. This problem is important not only for understanding the non-linear behavior of baryonic gas, but also for the theory of a Burgers' fluid.

The paper is organized into the following sections: $\S 2$ reviews the derivation of the Burgers' equations and its basic predictions for the baryonic gas. $\S 3$ gives a brief description of the hydrodynamic cosmological simulations. $\S 4$ gives the statistical analysis of the velocity field of the baryonic gas. Finally, in $\S 5$, we will present our conclusions followed by a discussion of the results. The velocity field in the discrete wavelet (DWT) representation is given in the Appendix.

\section{Cosmic baryonic gas velocity field}

\subsection{Burgers' equation for cosmic baryonic gas}

The derivation of Burgers' equation for cosmic baryonic gas has been addressed in Feng et al (2004), He et al. (2004) and Pando et al. (2004). In summary, the baryonic gas is assumed to be an ideal fluid satisfying the hydrodynamic equations

$$
\begin{gathered}
\frac{\partial \delta}{\partial t}+\frac{1}{a} \nabla \cdot(1+\delta) \mathbf{v}=0 \\
\frac{\partial a \mathbf{v}}{\partial t}+(\mathbf{v} \cdot \nabla) \mathbf{v}=-\frac{1}{\rho} \nabla p-\nabla \phi \\
\frac{\partial \mathcal{E}}{\partial t}+5 \frac{\dot{a}}{a} \mathcal{E}+\frac{1}{a} \nabla \cdot(\mathcal{E} \mathbf{v})=-\frac{1}{a} \nabla \cdot(p \mathbf{v})-\frac{1}{a} \rho_{i g m} \mathbf{v} \cdot \nabla \phi-\Lambda_{r a d}
\end{gathered}
$$

where $\rho, \mathbf{v}, \mathcal{E}$ and $p$ are, respectively, the mass density, peculiar velocity, energy density and pressure of the gas. The term $\Lambda_{\text {rad }}$ in Eq.(3) is given by radiative heating and cooling per unit volume of the baryonic gas. The gravitational potential $\phi$ in eqs.(2) and (3) is given by

$$
\nabla^{2} \phi=4 \pi G a^{2} \bar{\rho}_{d m} \delta_{d m},
$$

where $\bar{\rho}_{d m}(t)$ and $\delta_{d m}$, are respectively, the mean mass density and density contrast of the perturbations of dark matter. Here we assume that the gravitational potential $\phi$ is only given by the dark matter mass perturbation. 
To sketch the evolution of gravitational clustering we only consider the case where all thermal processes are approximated by the polytropic relations $p \propto \rho^{\gamma}, T \propto \rho^{\gamma-1}$, or $T=T_{0}(1+\delta)^{\gamma-1}$, where $\delta(\mathbf{x}, t)=[\rho(\mathbf{x}, t)-\bar{\rho}(t)] / \bar{\rho}(t)$ is the baryon mass density perturbation. The momentum equation is then

$$
\frac{\partial a \mathbf{v}}{\partial t}+(\mathbf{v} \cdot \nabla) \mathbf{v}=-\frac{\gamma k_{B} T}{\mu m_{p}} \frac{\nabla \delta}{(1+\delta)}-\nabla \phi
$$

where the parameter $\mu$ is the mean molecular weight of the IGM particles, and $m_{p}$ the proton mass. Using the linear approximation for $\delta$ in the temperature-dependent term of eq.(5), we have

$$
\frac{\partial a \mathbf{v}}{\partial t}+(\mathbf{v} \cdot \nabla) \mathbf{v}=-\frac{\nu}{a} \nabla^{2} \mathbf{v}-\nabla \phi
$$

where the coefficient $\nu$ is given by

$$
\nu=\frac{\gamma k_{B} T_{0}}{\mu m_{p}(d \ln D(t) / d t)},
$$

in which $D(t)$ describes the linear growth behavior. The term with $\nu$ in eq.(6) describes a diffusion characterized by the Jeans length $k_{J}^{2}=$ $\left(a^{2} / t^{2}\right)\left(\mu m_{p} / \gamma k_{B} T_{0}\right)$.

To understand cosmic large scale structure formation, only the growth modes are of interest. In this case, the velocity field of the perturbations is irrotational and we can define a velocity potential by

$$
\mathbf{v}=-\frac{1}{a} \nabla \varphi
$$

Substituting eq.(8) into eq.(6), we have

$$
\frac{\partial \varphi}{\partial t}-\frac{1}{2 a^{2}}(\nabla \varphi)^{2}-\frac{\nu}{a^{2}} \nabla^{2} \varphi=\phi
$$

Equation (9) is the stochastic-force-driven Burgers' equations or the KPZ equations (Kardar, Parisi \& Zhang 1986; Barabási \& Stanley 1995). This is the simplest nonlinear Langevin equation for growth modes. The second term on the l.h.s. is from convection which is the lowest-order term for nonlinear growth of clustering. The third on the 1.h.s. describes relaxation of the clustering by diffusion. The term on the r.h.s is the gravitational potential of dark matter, which provides the initially random perturbations. Clustered structures will develop from the initial seeds via the competition of the convection and diffusion. The Burgers' equation has been extensively applied in the non-linear dynamics of growing structures from a smoothed surface driven by random forces (e.g. Vicsek 1992). Eq. (9) shows that structure formation of baryonic gas in the universe can be thought of as the evolution of surface roughening, i.e., an initially smoothed distribution evolves into a wrinkled one.

\subsection{Baryonic gas as a Burgers' fluid}

Much theoretical work on Burgers fluids has been done in the hydrodynamic community in the past decade (Polyakov 1995; Bouchaud et al. 1995; Yakhot 1998; Lässig, 2000; Davoudi et al. 2001). Here we summarize the salient points necessary for testing whether cosmic baryonic gas behaves as Burgers' fluid.

In the Burgers' equation, there are two scales: the dissipation length or the Jeans length $1 / k_{J}$ and the correlation length of the random force, $r_{c}$. The intensity of the random force $\phi$ can be quantified by the density contrast of dark matter $\delta_{d m}$. The basic feature of the Burgers equation is that turbulence will developed in the fluid if the following condition holds (e.g. Lässig 2000; Feng et al 2003)

$$
\left(k_{J} r_{c}\right)^{2 / 3}\left\langle\delta_{d m}^{2}\right\rangle^{1 / 3}>1
$$

This condition corresponds to the system being in the non-linear regime of the dark matter field. In linear regime, $\delta_{d m} \ll 1$, and therefore, perturbations on scale $r_{c}>1 / k_{J}$ will not cause Burgers' turbulence in the IGM. In the nonlinear regime, $\delta_{d m}>1$, and Burgers' turbulence on scales larger the Jeans length can develop. Since the dark matter field becomes nonlinear first on small scales and then on large scales, one can expect that Burgers' turbulence in IGM develops from small to large scales

Burgers turbulence is qualitatively different from turbulence described by the Navier-Stokes equations. The latter generally consists of vortices on various scales, while the former is a collection of shocks. These features arise because for growth modes, the fluid is potential and the velocity field is irrotational. If $\nu \rightarrow 0$, the velocity field in the Burger prescription acquires singularities due to the discontinuities caused by strong shocks. The nonlinear feature of the baryonic gas velocity field with fully developed Burgers turbulence can be 
understood as a field consisting of these shocks (Lässig 2000).

Since dark matter is not affected by Burgers turbulence, the IGM velocity field dynamically decouples from the dark matter field on scales larger than the Jeans length when Burgers turbulence develops. Jeans diffusion will also lead to the decoupling between the velocity fields of IGM $\mathbf{v}$ and dark matter $\mathbf{v}_{d m}$. However, the decoupling given by the Jeans diffusion is very different from that given by the Burgers turbulence shocks. For the former, the distribution $\mathbf{v}$ and $\Delta \mathbf{v} \equiv \mathbf{v}(\mathbf{x}+\mathbf{r})-\mathbf{v}(\mathbf{x}-\mathbf{r})$ is symmetric with respect to the transformation $\mathbf{v}_{d m} \rightarrow-\mathbf{v}_{d m}$, i.e., the velocity PDF with $\Delta \mathbf{v} \cdot \mathbf{v}_{d m} /\left|\mathbf{v}_{d m}\right|>0$ (acceleration in the direction of $\mathbf{v}_{d m} /\left|\mathbf{v}_{d m}\right|$ ) is the same as the PDF with $\Delta \mathbf{v} \cdot \mathbf{v}_{d m} /\left|\mathbf{v}_{d m}\right|<0$ (deceleration in the direction of $\left.\mathbf{v}_{d m} /\left|\mathbf{v}_{d m}\right|\right)$. For Burgers turbulence, the shocks consist of an acceleration ramp followed by a rapid deceleration. Therefore, the IGM is not symmetric between the sections accelerating and decelerating. Since the acceleration is due to the gravity of dark matter, the acceleration is generally in the direction of $\mathbf{v}_{d m}$. Thus, the IGM velocity field $\mathbf{v}$ is asymmetric with respect to the transformation $\mathbf{v}_{d m} \rightarrow-\mathbf{v}_{d m}$. The PDF of $\Delta \mathbf{v}$ with $\Delta \mathbf{v} \cdot \mathbf{v}_{d m} /\left|\mathbf{v}_{d m}\right|>0$ will not be the same as the PDF of $\Delta \mathbf{v}$ with $\Delta \mathbf{v} \cdot \mathbf{v}_{d m} /\left|\mathbf{v}_{d m}\right|<0$.

If $\nu=0$, Burgers equation (9) is scale free when the random force $\phi$ is scale-free. In this case, the velocity field of the Burgers fluid is selfsimilar and the PDF of the velocity difference $\Delta \mathbf{v}$ is scale-invariant. The velocity difference can be effectively measured by the longitudinal velocity difference defined as $\Delta v_{r} \equiv v_{\|}\left(\mathbf{r}_{1}, t\right)-v_{\|}\left(\mathbf{r}_{2}, t\right)$, where $v_{\|} \equiv \mathbf{v} \cdot\left(\mathbf{r}_{1}-\mathbf{r}_{2}\right) / r$ and $r=\left|\mathbf{r}_{1}-\mathbf{r}_{2}\right| . \Delta v_{r}$ measures the acceleration and deceleration along direction $\left.\mathbf{r}_{1}-\mathbf{r}_{2}\right) / r$. A detailed analysis shows that for a self-similar velocity field, the PDF of $\Delta v_{r}$ is (e.g. Davoudi et al 2001)

$$
P\left(\Delta v_{r}, r\right)=\frac{1}{r^{y}} F\left(\frac{\Delta v_{r}}{r^{y}}\right),
$$

where parameter $y$ depends on the statistical properties of the random force.

Summarizing, if the IGM can be described as a Burgers' fluid, its velocity field should show the following properties: 1.) the peculiar velocity of the IGM at a given point will generally be lower than that of dark matter at the same point, 2.) the PDF of $\Delta \mathbf{v}_{r} \cdot \mathbf{v}_{d m} /\left|\mathbf{v}_{d m}\right|>0$ will not be the same as the PDF of $\Delta \mathbf{v}_{r} \cdot \mathbf{v}_{d m} /\left|\mathbf{v}_{d m}\right|<0$, and 3.) The PDF of $\Delta \mathbf{v}_{r}$ scales. We will test for these properties in the following sections.

\section{Cosmological hydrodynamic simula- tions}

Burgers equation (9) is obtained under the assumptions that 1) decaying modes are ignored; 2) the cooling and heating of the IGM are ignored and replaced by a polytropic equation of state, and 3) the diffusion term is given in the linear approximation. Moreover, the driving force in the Burgers equation for the baryonic gas comes only from the dark matter gravity, which itself undergoes evolution. It is not at all clear that the features of a Burgers fluid occur in the IGM for which cooling and heating processes are essential. To answer this question, we determine whether samples produced by a fully cosmological hydrodynamical simulation have the properties listed at the end of last section.

\subsection{The WENO algorithm}

In order to capture the Burgers fluid features, simulations need to be effective in capturing shocks and discontinuities. Hence we do not use a Lagrangian approach such as the smoothed particle hydrodynamic (SPH) algorithm. The reason is the SPH scheme does not handle shocks or discontinuities well because the nature of $\mathrm{SPH}$ is to smooth the fields (e.g. Borve, Omang, \& Trulsen, 2001; Omang, Borve, \& Trulsen 2003.) Consequently, Burgers fluid features are overlooked.

We instead take a Eulerian approach. A wellknown problem with the Eulerian algorithm are the unphysical oscillations near a discontinuity. An effective method to reduce the spurious oscillations is given by designed limiters, such as the Total-Variation Diminishing (TVD) method (Harten 1987 et al.) or the piecewise parabolic method (PPM) (Collella \& Woodward 1984). However, the accuracy in the TVD method degenerates to first order near smooth extrema (Godlewski \& Raviart 1996). This problem will cause errors in calculating the temperature and entropy changes because they are determined by the difference of the thermal energy $P /(\gamma-1)$ on two sides of the shock. When the Mach number 
of gas is high, the thermal energy $P /(\gamma-1)$ is very small compared to the kinetic energy $\rho \mathbf{v}^{2} / 2$. To overcome this problem, the Essentially NonOscillatory (ENO) algorithm and Weighted Essentially Non-Oscillatory (WENO) were proposed (Harten et al. 1987; Shu 1998; Fedkiw, Sapiro \& Shu 2003; Shu 2003). They can simultaneously provide a high order precision for both the smooth part of the solution and the sharp shock transitions(Liu, Osher, \& Chan 1994; Jiang \& Shu 1996).

WENO has been successfully applied to hydrodynamic problems containing shocks and complex structures. WENO has also been used to study astrophysical hydrodynamics, including stellar atmospheres (del Zanna, Velli \& Londrillo 1994 ), high Reynolds number compressible flows with supernova (Zhang et al. 2003), and high Mach number astrophysical jets (Carrillo et al. 2003). In the context of cosmological applications, WENO has proved to be especially adept at handling the Burgers' equation (Shu 1999). Recently, a hybrid hydrodynamic/N-body code based on the WENO scheme was developed. It has passed typical reliability tests including the Sedov blast wave and the formation of the Zeldovich pancake (Feng, Shu \& Zhang 2004). This code has successfully produced QSO Ly $\alpha$ transmitted flux samples, including the high resolution sample HP1700+6416 (Feng, Pando \& Fang 2003). The statistical features of these samples are in good agreement with observed features not only on second order measures, like the power spectrum, but also up to orders as high as eighth for the intermittent behavior.

\subsection{Samples}

For the purpose of this paper, we run the hybrid $N$-body/hydrodynamic code to trace the cosmic evolution of the coupled system of both dark matter and baryonic gas in a flat low density CDM model $(\Lambda \mathrm{CDM})$, which is specified by the cosmological parameters $\left(\Omega_{m}, \Omega_{\Lambda}, h, \sigma_{8}, \Omega_{b}\right)=$ $(0.3,0.7,0.7,0.9,0.026)$. The baryon fraction is fixed using the constraint from primordial nucleosynthesis as $\Omega_{b}=0.0125 h^{-2}$ (Olive et al. 1991). The value of $\Omega_{b}$ is lower than the current result from WMAP. However, since we we need to refer our previous results (Feng et. al. 2003, Pando et. al. 2004, He et. al. 2004), which are based on the simulation samples produced before WMAP, we use the older value. The baryon fraction is not a parameter that enters into Burgers' equation, and therefore Burgers' fluids features do not seriously depend on the baryon fraction once the gravitation of the baryon gas is negligible in comparison to that of dark matter.

The simulations are performed in a periodic, cubic box of size $L^{3}=25 \mathrm{~h}^{-1} \mathrm{Mpc}$ with a $192^{3}$ grid and an equal number of dark matter particles. The size of the grid is $25 / 192=33 / 2^{8}=0.129 \mathrm{~h}^{-1}$ Mpc. The thickness of the Burgers' shock is on the order of the dissipation length, i.e., the Jeans length, which is in the range $\sim 0.1-0.3 \mathrm{~h}^{-1} \mathrm{Mpc}$ for redshifts $z<4$ (Bi et al. 2003). Therefore, the resolution of our simulation is enough to capture shocks. For strong shocks, the thickness of the shock is less than the dissipation length. However, strong shocks are generally associated with high temperature regions (i.e. $\gg 10^{4} \mathrm{~K}$ ), and the local Jeans length is larger. Therefore, the resolution is still less than the Jeans length. In Feng et al 2003, we used two samples with different resolution. The major statistical results were found to be independent of the resolutions.

The simulations start at a redshift $z=49$ and the results are recorded at redshifts $z=6.0,4.0$, $3.0,2.0,1.0,0.5$ and 0.0 . The time step is chosen by the minimum value among the following three time scales. The first is from the Courant condition. The second time scale is imposed by cosmic expansion which requires that $\Delta a / a<0.02$ within a single time step. The last time scale comes from the requirement that a particle moves not more than a fixed fraction of the cell size.

Atomic processes, including ionization, radiative cooling and heating in a plasma of hydrogen and helium of primordial composition $(X=0.76$, $Y=0.24)$ are modeled in the same way as in Bahcall \& Cen (1992). Processes such as star formation, feedback due to SN and AGN activities have not yet been taken into account. A uniform UV-background of ionizing photons is assumed to have a power-law spectrum of the form $J(\nu)=$ $J_{21} \times 10^{-21}\left(\nu / \nu_{H I}\right)^{-\alpha} \mathrm{erg} \mathrm{s}^{-1} \mathrm{~cm}^{-2} \mathrm{sr}^{-1} \mathrm{~Hz}^{-1}$, with parameter $J_{21}=1.0$ and $\alpha=1$. The photoionizing flux is suddenly switched on at $z>10$ to heat the gas and reionize the universe.

For our work here, we randomly sampled 192 data points along 500 one-dimensional directions 
$\left(500 \times 192=9.6 \times 10^{4}\right.$ total points. $)$ At each point, the mass density $\rho_{d m}$ and peculiar velocity component along the line of sight $v_{d m}$ of dark matter, and mass density $\rho_{\text {igm }}$, peculiar velocity component along the line of sight $v$ and temperature $T$ of the baryonic gas are recorded. At times during our analysis it will be necessarry to smooth the distributions on scales of $33 / 2^{j} \mathrm{~h}^{-1} \mathrm{Mpc}$, where $j=3,4,5,6,7$.

\section{Statistical analysis of velocity fields}

\subsection{Relation between the IGM and dark matter velocity fields}

In the linear growth regime, the IGM velocity follows the dark matter velocity point by point, i.e., $\mathbf{v}(\mathbf{x}, t)=\mathbf{v}_{d m}(\mathbf{x}, t)$ (Bi, Börner, \& Chu 1993; Fang et al. 1993; Bi 1993, Nusser 2000.) Burgers' turbulence will lead to a deviation of $\mathbf{v}(\mathbf{x}, t)$ from $\mathbf{v}_{d m}(\mathbf{x}, t)$. Figure 1 gives the relation between the velocities of the IGM and dark matter at each grid point of the samples at redshifts $6,4,2,1,0.5$ and 0 , where the data is smoothed on scale $33 / 2^{7}$ $\mathrm{h}^{-1}$ Mpc. As expected, the data points are scattered around the diagonal line $v=v_{d m}$. At high redshift $z=6$, the scatter is smaller, reflecting the fact that the fields are still in the quasi-linear regime. At $z<1$, the scatter becomes significant. The scatter at low redshift cannot be explained by the Jeans diffusion or random noise as Figure 1 shows a remarkable butterfly-like configuration, i.e., $v$ mostly lies $0<v<v_{d m}$ if $v_{d m}>0$, while it lies $0>v>v_{d m}$ when $v_{d m}<0$. In other words, the peculiar velocity of IGM $|v|$ is generally less than $\left|v_{d m}\right|$. As mentioned in $\S 2.2$, shocks naturally yield the butterfly-like configuration because shocks always lead to a deceleration of the gas with respect to the underlying dark matter.

Figure 1 also shows an envelope of the scatter at about $v \simeq v_{d m} / 4$. This means, the velocity decrease due to shocks generally is not larger than a factor of 4 . This factor is consistent with the shock theory of a polytropic gas (Landau \& Lifshitz 1959). The strongest shocks of a polytropic gas lead to an decrease of the velocity by a factor of $\sim(\gamma-1) /(\gamma+1)$. For these samples the value of $\gamma$ is found to be $5 / 3$ (He at al. 2004). Therefore, the velocity decrease should not be larger than a factor $(\gamma+1) /(\gamma-1)=4$.

Figure 2 shows the relation beteen $v$ and $v_{d m}$ at $z=0$ for the density ranges: (1) low density $\rho_{d m}<1$, (2) moderate density $1<\rho_{d m}<5$, (3) high density $5<\rho_{d m}$. We see that even in the low and moderate density region, the scatter of $v$ is still butterfly-like. This means that the velocity fields are dominated by shock structures not only around massive halos, but also in moderate density areas. Burgers turbulence consists of shocks regardless of whether the density is high.

Figure 3 shows again the relation between $v$ and $v_{d m}$, but $v$ and $v_{d m}$ are now smoothed on scales $d=33.3 / 2^{j} \mathrm{~h}^{-1} \mathrm{Mpc}$. We see that the butterfly feature is weaker with increasingly smooth scales. However, this does not mean that the deviation of $v$ from $v_{d m}$ is smaller. On the contrary, the distribution of $v$ still substantially deviates from the diagonal line for smoothing scales from 2 to $8 \mathrm{~h}^{-1}$ Mpc. This happens because when the smoothing scale is larger than the mean separation of strong shocks, the strong shocks are localized within each area. Consequently, the mean velocity in all regions is always dominated by these strong shocks.

This point can be more clearly seen in Figure 4, which plots the relation between $v$ and $v_{d m}$ at redshifts $6,4,2,1,0.5$, and 0 for the field smoothed on scales $d=8 \mathrm{~h}^{-1}$ Mpc. Figure 4 shows that at redshift 6 , the velocity distribution basically follows $v=v_{d m}$ with little scatter. As redshift decreases, the distribution of $v$ is always tightly correlated with $v_{d m}$, but the correlation between $v$ and $v_{d m}$ is not the diagonal line $v=v_{d m}$, but $v=s v_{d m}$ with the slope $s<1$. The parameter $s$ decreases with redshift, it is 0.9 at $z=6$, and 0.4 at $\mathrm{z}=0$. Essentially, $s$ is a measure of the effect of strong shocks at various redshift eras. Since Burgers' shocks are related to the formation of cusps in the underlying dark matter density field, the shock growth is parallel with the development of non-linear clustering. Therefore, the deviation of s from 1 is bigger for lower redshifts.

\subsection{Relations of velocity-density and velocity- temperature}

We next analyze the relation between mass density and velocity. Figure 5 shows $v$ vs. $\rho_{d m}, v$ vs. $\rho_{i g m}, v_{d m}$ vs. $\rho_{d m}$ and $v_{d m}$ vs. $\rho_{i g m}$ at each grid point of the samples at redshift $z=0$. At a given $\rho_{i g m}$ or $\rho_{d m}$, the scatter of $v_{d m}$ is much larger than $v$. 
More interesting is the qualitative difference between the scatter of $v$ and $v_{d m}$. The scatter of the IGM velocity $v$ has a clear $\rho_{d m}$ (or $\rho_{i g m}$ )dependent envelope: the smaller the mass densities, the smaller the scatter. This is because the IGM flow is potential. In low density areas, the gravitational potential does not vary significantly. Bernoulli's equation predicts that the greatest possible value of fluid velocity for a potential flow is proportional to the speed of sound (Landau \& Lifshitz 1959). Since the speed of sound generally increases with gas density, the maximum IGM velocity, i.e., the velocity at the $v-\rho_{\text {igm }}$ envelope, increases with $\rho_{\text {igm }}$. Although $\rho_{i g m}$ and $\rho_{d m}$ are not the same point-by-point, a large $\rho_{d m}$ on average corresponds to large $\rho$ (Pando et al 2004). Therefore, the relation $v$ vs. $\rho_{d m}$ shows a similar envelope. On the other hand, the dark matter velocity $v_{d m}$ is not affected by the IGM shocks and is not constrained by the speed of sound of the IGM. There is no density-dependent envelope for $v_{d m}$.

Figure 6 plots the relation between the IGM velocity $v$ and temperature $T$ at each grid point of the samples at redshift $z=0$. The scatter of the IGM velocity $v$ with respect to $T$ also has a clear envelope: the smaller the temperature, the smaller the scatter. This envelope develops for the same reason as above: the IGM velocity cannot be much larger than the speed sound. However, figure 6 shows that the envelope increases with $T$ quickly at $T \leq 3,000 \mathrm{~K}$, but slowly at $T \geq 3,000 \mathrm{~K}$. This is probably because most hydrogen atoms are neutral at $T \leq 3,000 \mathrm{~K}$ and ionized at $T \geq 3,000 \mathrm{~K}$. On the other hand, the velocity of dark matter $v_{d m}$ is not subject to this constrains. Therefore, there is no envelope in the $v_{d m}-T$ scatter distribution.

It should be pointed out that the envelopes shown in Figures 5 and 6 significantly deviate from the usual relation $v \propto \sqrt{(T)}$ for the speed of sound. The reason is that the baryon gas exists in different regions of $T--\rho$ space. That is, the baryon gas is in multiple phases. The multiple phases of cosmic baryon gas has been addressed in He, Feng \& Fang (2004) where it is shown that the $T-\rho$, and $S$ (entropy) $-T$ and $S-\rho$ relations are significantly multiply phased either at low or high temperature or density. The deviation from the usual relation of the velocity and temperature shown in Figs 7 and 8 is evidence that the baryon gas is substantially multiple phased.

\subsection{Asymmetry of the PDF of velocity difference}

As mentioned in $\S 2.2$, the features of a Burgers fluid are effectively described by the statistics of the longitudinal velocity difference $\Delta v_{r}(\mathbf{x})=$ $v_{\|}[\mathbf{x}+(\mathbf{r} / 2)]-v_{\|}[\mathbf{x}-(\mathbf{r} / 2)]$. This quantity describes the velocity difference at physical position $\mathbf{x}$ and on distance $r=|\mathbf{r}|$. That is, $\Delta v_{r}(\mathbf{x})>0$ corresponds to an acceleration event in direction $\mathbf{r}$, while $\Delta v_{r}(\mathbf{x})<0$ is a deceleration event. More useful is to define the acceleration and deceleration with respect to the velocity direction of the underlying dark matter velocity field. For one-dimensional fields, the events of $\Delta v_{r}(x) v_{d m}(x) /\left|v_{d m}(x)\right|>0$ correspond to an acceleration with respect to the dark matter velocity field, while $\Delta v_{r}(x) v_{d m}(x) /\left|v_{d m}(x)\right|<0$ the deceleration events.

The velocity difference $v(x+r)-v(x-r)$ contains position $x$ and scale $r$. It is convenient to measure $\Delta v_{r}(x)$ by the discrete wavelet transform (DWT) $\Delta v_{j, l}$ (see Appendix). Moreover, the DWT modes are orthogonal and effectively avoid false correlations and scalings. The smoothing can also be done with the DWT, as the scaling functions on various scales are window functions on that scale. For our 1-dimensional samples, the DWT variable $\Delta v_{j, l}$ (see Appendix) describes the velocity difference at the physical position of cell $l$ and on distance $r=33.3 / 2^{j} \mathrm{~h}^{-1} \mathrm{Mpc}$. With the DWT variables, the acceleration and deceleration events with respect to the dark matter field are given by $\Delta v_{j, l}\left(v_{j, l} /\left|v_{j, l}\right|\right)_{d m}$.

We showed in $\S 4.1$ that the IGM velocity is generally lower than the dark matter velocity because of shocks. Shocks cause a decrease in the velocity in the direction of the gas flow $v$. This means the $\mathrm{PDF}$ of $\Delta v_{j, l}$ will be asymmetric between acceleration and deceleration events. Figure 7 plots the PDFs of $\Delta v_{j, l}$ for samples at $z=0$ and at smoothing scales $j=3,4,5$ and 6 . It is clear that the PDFs of acceleration and deceleration branches of the distribution are asymmetric. For $j \leq 5$, the PDFs of deceleration events generally have a longer tail than that of acceleration events, i.e., there are more big deceleration events than acceleration events. Using a $K-S$ test, we find that the probability the the acceleration and deceleration 
branches are drawn from the same distribution is 0.048 for $j=4$, and 0.28 for $j=3$. Therefore, the asymmetry is weaker on larger scale.

Generally, the asymmetric long tail is significant at $\left|\Delta v_{j, l}\right|>20 \mathrm{~km} / \mathrm{s}$. For $\left|\Delta v_{j, l}\left(v_{j, l} /\left|v_{j, l}\right|\right)_{d m}\right| \leq$ $20 \mathrm{~km} / \mathrm{s}$, the asymmetry is weaker, because the velocity difference caused by the Jeans diffusion is equal to about $20-30 \mathrm{~km} / \mathrm{s}$. This is also the reason that the asymmetry almost disappeared on scales equal to and less that $0.5 \mathrm{~h}^{-1} \mathrm{Mpc}$, or $j=6$. The K-S probability is 0.15 for $j=5$ and 0.79 for $j=6$. The thickness of shocks due to the Jeans diffusion is on order $\nu /\left\langle v^{2}\right\rangle^{1 / 2}$, where $\left\langle v^{2}\right\rangle^{1 / 2}$ is the rms of velocity field. Therefore, the velocity difference $\Delta v_{r}(x)$ with $r$ less than $\nu /\left\langle v^{2}\right\rangle^{1 / 2}$ will not be sensitive to the shock discontinuity. Moreover, the 1-dimensional samples will also weaken the asymmetry. Since $v_{d m}$ is a projection of the 3 -dimensional $\mathbf{v}_{d m}$, the asymmetry will be missed for events perpendicular to the direction of 1-dimension sampling.

Figure 8 shows the PDFs of $\Delta v_{j, l}$ on scale $j=$ 4 , but for redshifts $z=0,1.0,2.0$ and 4.0. This figure shows that the PDFs is already asymmetric at $z=4$. The number of large deceleration events is higher for lower redshifts. This yields the slope $s<1$ in Figure 4 that is smaller for lower redshifts.

\subsection{Scaling of the $\Delta v_{j, l}$ PDFs}

An important property of the Burgers fluid is the self-similarity of the velocity field as given by eq.(11). A simplified version of eq.(11) can be obtained as follows. For the IGM field, the velocity gradient is $\left(\Delta v_{r}\right) / r$. If the field of the Burgers' turbulence shocks is self-similar, the PDF of $\left(\Delta v_{r}\right) / r, F\left[\left(\Delta v_{r}\right) / r\right]$, should be scale-invariant. In this case, the $\mathrm{PDF}$ of $\Delta v_{r}, P\left(\Delta v_{r}\right)$, is given by $P\left(\Delta v_{r}\right) d\left(\Delta v_{r}\right)=F\left[\left(\Delta v_{r}\right) / r\right] d\left(\Delta v_{r} / r\right)$. Thus, we have $P\left(\Delta v_{r}\right)=(1 / r) F\left[\left(\Delta v_{r} / r\right)\right.$. This is the PDF of eq.(11) with $y=1$.

Using DWT variables and noting that the physical scale $r$ is given by index $j$ as $r=33.33 / 2^{j}$, eq.(11) can be rewritten as

$$
P\left(\Delta v_{j, l}\right)=2^{y j} F\left(2^{y j} \Delta v_{j, l}\right) .
$$

Eq.(12) requires that the dynamics of the velocity difference $\Delta v_{j, l}$ be scale-free. It is not immediately clear whether the IGM velocity field is really scale-invariant because the IGM dynamical equations (1) - (4) contain many physical scales, such as the Jeans scale; the scales related to cooling and heating, etc., and these are not necessarily scale free.

We tried to fit the distribution $P\left(\Delta v_{j, l}\right)$ with a scale-invariant function like (12). The first result is shown in Figure 9, which gives the $P\left(\Delta v_{j, l}\right)$ against $\Delta v_{j, l}$ for the deceleration branch for samples at $z=0$. We found the best scaling function is

$$
P\left(\Delta v_{j, l}\right)=0.012 \times 2^{0.7 j} \exp \left[-\left(2^{0.7 j} \frac{\Delta v_{j, l}}{50 \mathrm{~km} / \mathrm{s}}\right)^{0.75}\right]
$$

The PDF eq.(13) is consistent with eq.(12). The quantity 0.012 is given by the overall normalization and can be absorbed by a unit transformation. The scaling behavior of the PDF depends on the two scaling factors $2^{0.7 j}$ and $\left(2^{0.7 j}\right)^{0.75}$, and therefore, the scaling is given by two parameters: $y=0.70$, and the index 0.75 . The factor $50 \mathrm{~km} / \mathrm{s}$ makes the exponential dimensionless, but is not involved in the scaling and has no $j$ dependence. The scaling function (13) excellently fits $\operatorname{PDF} P\left(\Delta v_{j, l}\right)$ on scales $j=2$ - 7 , i.e., from the highly nonlinear scale $0.26 \mathrm{~h}^{-1} \mathrm{Mpc}$ to the weak nonlinear scale $8 \mathrm{~h}^{-1} \mathrm{Mpc}$.

Figure 10 is similar to Figure 9, but for the acceleration branch. The scaling function is now

$$
P\left(\Delta v_{j, l}\right)=0.028 \times 2^{0.47 j} \exp \left[-\left(2^{0.47 j} \frac{\Delta v_{j, l}}{28 \mathrm{~km} / \mathrm{s}}\right)^{0.75}\right]
$$

Similar to eq.(13), the scaling behavior of eq.(14) depends on two parameters: $y=0.47$, and the in$\operatorname{dex} 0.75$. The factor $28 \mathrm{~km} / \mathrm{s}$ makes the exponential dimensionless. Eq.(14) also gives a very good fit to the PDFs from $j=2$ to $j=6$. The PDFs at $j=7$ shows small deviation from eq.(14). This is probably because the scale $j=7$, or $0.26 \mathrm{~h}^{-1}$ $\mathrm{Mpc}$ is comparable with the Jeans length. The parameter $y=0.47$ in eq.(14) is significantly different than value given in Eq.(13). This difference indicates that although both the the acceleration and deceleration branches scale, the scaling behavior is not symmetric between the acceleration and deceleration branches.

Both functions (13) and (14) are consistent with eq.(12). Burgers' equation predicts that the acceleration and deceleration branches will have different PDFs. All these results show that the scale in- 
variant behavior of IGM velocity field is the same as a Burgers' fluid. According to the general theory of Burgers' fluids (Davoudi et al 2001), the parameter $y$ should be dependent on the statistical property of the random force $\phi$ and the relation cannot be found analytically. The simulation box of size $L^{3}=25 \mathrm{~h}^{-1} \mathrm{Mpc}$ is not much larger than $8 \mathrm{~h}^{-1} \mathrm{Mpc}$, but the scaling shown in Figures 9 and 10 still gives a good fit on scale of $8 \mathrm{~h}^{-1} \mathrm{Mpc}$. The reason for this is the use of the velocity difference which suppresses the contribution of long wave length perterbations.

\section{Discussions and conclusions}

The initial mass density fluctuations of the universe are probably Gaussian with a scale-free power spectrum. Therefore, in the linear regime the dark matter and IGM mass and velocity fields are self-similar. In the non-linear regime, the dynamical behavior of the dark matter and IGM are very different even though they are coupled via gravity. Since the dynamics of the dark matter is governed only by the gravitational interactions which do not have a preferred scale, the dark matter should undergo self-similar evolution even in the non-linear regime. The evolution of the IGM seems to be much more complicated because it sensitively depends on thermal, radiative and other non-gravitational processes. Consequently, the properties of the Burgers' equation of the dark matter field cannot simply be transferred to the IGM (He et al. 2004.)

Nevertheless, we showed that the main dynamical feature of the IGM velocity field can be explained as a Burgers fluid. The IGM samples, produced by a full hydrodynamic simulation in which thermal processes are involved, show all features of a Burgers fluid. This is probably because the scales considered are larger than the Jeans length, but smaller than the scale at which the nonlinear evolution is onset. In this range the IGM is nonlinear, but not sensitive to the scales of thermal processes. Therefore, it can also be approximately described as a Burgers' fluid.

However, as a Burgers' fluid, the IGM velocity field is not a simple mirror of the underlying dark matter velocity field. For instance the PDFs of the velocity difference of dark matter is lognormal (Yang et al. 2001) and significantly different from eqs.(13) and (14). The PDF of the velocity difference of dark matter is symmetric between the acceleration and deceleration branches with respect to the velocity field of dark matter, but asymmetric for the IGM. All these differences come from shocks which do not develop in the dark matter. Non-linear evolution will inevitably lead to a dynamical break of the similarity between the dark matter and gas.

Ping He acknowledges support from the World Laboratory scholarship. LLF acknowledges support from the National Science Foundation of China (NSFC) and National Key Basic Research Science Foundation. This work is partially supported by the National Natural Science Foundation of China (10025313) and the National Key Basic Research Science Foundation of China (NKBRSF G19990752). 


\section{A. The DWT variables of velocity field}

For the details of the mathematical properties of the DWT refer to Mallat (1989a,b), Meyer (1992), Daubechies (1992), and for physical applications, refer to Fang \& Thews (1998). For us here, the most important properties are 1.) orthogonality, 2.) completeness, and 3.) locality in both scale $(r)$ and physical position $(x)$. Wavelets with compactly supported basis are an excellent means to analyze the velocity fields. Among the compactly supported orthogonal wavelets, the Daubechies family of wavelets are easy to implement.

To simplify the notation, we consider a 1-D velocity field $v(x)$ on spatial range $L$. It is straightforward to generalize to 3 -D fields. In DWT analysis, the space $L$ is chopped into $2^{j}$ segments labeled by $l=0,1, \ldots 2^{j}-1$. Each of the segments has size $L / 2^{j}$. The index $j$ is a positive integer which represents scale $L / 2^{j}$. The index $l$ gives position and corresponds to spatial range $l L / 2^{j}<x<(l+1) L / 2^{j}$.

DWT analysis uses two functions, the scaling functions $\phi_{j, l}(x)=\left(2^{j} / L\right)^{1 / 2} \phi\left(2^{j} / L-l\right)$, and wavelets $\psi_{j, l}(x)=\left(2^{j} / L\right)^{1 / 2} \psi\left(2^{j} / L-l\right)$. The scaling functions play the role of window function. They are used to calculate the mean field in the segment $l$. The wavelets $\psi_{j, l}(x)$ capture the difference between the mean fields at space ranges $l L / 2^{j}<x<(l+1 / 2) L / 2^{j}$ and $(l+1 / 2) L / 2^{j}<x<(l+1) L / 2^{j}$.

The scaling functions and wavelets $\psi_{j, l}(x)$ satisfy the orthogonal relations

$$
\begin{gathered}
\int \phi_{j, l}(x) \phi_{j, l^{\prime}}(x) d x=\delta_{l, l^{\prime}}, \\
\int \psi_{j, l}(x) \psi_{j^{\prime}, l^{\prime}}(x) d x=\delta_{j, j^{\prime}} \delta_{l, l^{\prime}}, \\
\int \phi_{j, l}(x) \psi_{j^{\prime}, l^{\prime}}(x) d x=0, \quad \text { if } j^{\prime} \geq j .
\end{gathered}
$$

With these properties, a 1-D random field $v(x)$ can be decomposed into

$$
v(x)=v^{j}(x)+\sum_{j^{\prime}=j}^{\infty} \sum_{l=0}^{2^{j^{\prime}}-1} \tilde{\epsilon}_{j^{\prime}, l} \psi_{j^{\prime}, l}(x),
$$

where

$$
v^{j}(x)=\sum_{l=0}^{2^{j}-1} \epsilon_{j, l} \phi_{j, l}(x) .
$$

The scaling function coefficient (SFC) $\epsilon_{j, l}$ and the wavelet function coefficient (WFC), $\tilde{\epsilon}_{j, l}$ are given by

$$
\epsilon_{j, l}=\int v(x) \phi_{j, l}(x) d x
$$

and

$$
\tilde{\epsilon}_{j, l}=\int v(x) \psi_{j, l}(x) d x
$$

respectively. The SFC $\epsilon_{j, l}$ measure the mean of $v(x)$ in the segment $l$, while the WFC $\tilde{\epsilon}_{j, l}$ measures the fluctuations (or difference) of field $v(x)$ at $l$ on scale $j$.

The first term on the r.h.s. of eq.(A4), $v^{j}(x)$, is the field $v(x)$ smoothed on the scale $j$, while the second term contains all information on scales $\geq j$. Because of the orthogonality, the decomposition between the scales of $<j$ (first term) and $\geq j$ (second term) in eq.(A4) is unambiguous.

Since scale $r$, and position $x$, correspond to, respectively, $j, l$, and $\phi_{j, l}\left(x^{\prime}\right)$ is window filter on scale $r$ at position $x$, the DWT variables of velocity field are given by

$$
v_{j, l}=\frac{\int v(x) \phi_{j, l}(x) d x}{\int \phi_{j, l}(x) d x}
$$


$v_{j, l}$ is the mean velocity in the spatial range $l L / 2^{j}<x<(l+1) L / 2^{j}$. Similarly, the DWT counterpart of the velocity difference is

$$
\Delta v_{j, l}=\frac{\int v(x) \psi_{j, l}(x) d x}{\int \phi_{j, l}(x) d x}
$$

$\Delta v_{j, l}$ is the difference between the mean velocities of spatial ranges $l L / 2^{j}<x<(l+1 / 2) L / 2^{j}$ and $(l+$ $1 / 2) L / 2^{j}<x<(l+1) L / 2^{j}$.

$v_{j, l}$ and $\Delta v_{j, l}$ are the variables of the velocity field $v(x)$ in the DWT representation. These variables give a complete description of the field $v(x)$ without a loss of information. The orthogonality of scaling functions and wavelets insure that the decomposition does not cause false correlation among these variables. 


\section{REFERENCES}

Bahcall, N.A., Cen, R. Y. 1992, ApJ, 398, L81

Balkovsky, E., Falkovich, G., Kolokolov, I. \& Lebedev, V. 1997, Phys. Rev. Letts. 78, 1452

Barabasi, A.L \& Santley, H.E., 1995, Fractal Concepts in Surface Growth, (Cambridge University Press).

Berera, A. \& Fang, L.Z. 1994, Phys. Rev. Letts. 72,458

Bi H.G. 1993, ApJ, 405, 479

Bi, H.G., Borner, G. \& Chu, Y. 1993, A\&A 405, 479

Bi, H.G., Fang, L.Z., Feng, L.L. \& Jing, Y.P. 2003, ApJ, 598, 1

Borve, S., Omang, M. \& Trulsen, J. 2001, ApJ, 56,82

Bouchaud, J.-P., Mézard, M. \& Parisi, G. 1995, Phys. Rev. E52, 3656

Carrillo, J.A., Gamba, I.M., Majorana, A. \& Shu, C.-W, 2003, Journal of Computational Electronics, 1, 365 .

Collella, P. \& Woodward, P.R. (1984), Journal of Computational Physics, 54, 174.

Daubechies I. 1992, Ten Lectures on Wavelets, (Philadelphia, SIAM)

Davoudi, J., Masoudi, A. A., Rahimi T., Rastegar, A., \& Shahbazi, F. (2001) Physical Review E, 63,056308

Del Zanna, L., Velli, L. \& Londrillo, P. 1994, A\&A 330, L1

Ettori, S., \& Fabian, A.C. 1999, MNRAS, 305, 834

Fang, L. Z, Bi, H., Xiang, S. \& Boerner, G. 1993, ApJ, 413, 477

Fang, L.Z. \& Thews, R. 1998, Wavelet in Physics, (World Scientific, Singapore)

Fedkiw, R.P., Sapiro, G., \& Shu, C.W. 2003, J. Comput. Phys., 185, 309

Feng, L.L, Pando, J., \& Fang, L.Z. 2003, ApJ, 587, 478

Feng, L.L, Shu, C.W., \& Zhang, M. 2004, ApJ, 612,1

Frisch, U., Bec, J. \& Villone, B. 1999, condmat/9912110
Godlewski, E., \& Raviart, P.A. 1996, Numerical approximation of hyperbolic systems of conservation laws, (Springer)

Gurarie, V. \& Migdal, A. 1996, Phys. Rev. E54, 4908

Gurbatov, S.N., Saichev, A.I. \& Shandarin, S.F. 1989, MNRAS, 236, 385

Harten, A., Engquist, B., Osher, S., \& Chakravart, S. (1987) Journal of Computational Physics, 71, 231.

He, P., Feng, L.L. \& Fang, L.Z. 2004, ApJ, 612, 14

He, P., Feng, L.L. \& Fang, L.Z. 2005, ApJ, in press

Jamkhedkar, P., Feng, L.L., Zheng, W., Kirkman, D., Tytler, D. \& Fang, L.Z. 2003 MNRAS, 343, 1110

Jamkhedkar, P., Zhan, H. \& Fang, L.Z. 2000, ApJ, 543, L1

Jiang, G. \& Shu, C.-W. (1996) Journal of Computational Physics, 126, 202.

Jones, B.T., 1999, MNRAS, 307, 376

Kardar, M., Parisi, G. \& Zhang, Y.C. 1986, Phys. Rev. Letts. 56, 889.

Landau, L. \& Lifshitz, M.L., (1959) Fluid Mechanics, (Pergamon Press, Oxford)

Lässig, M. 2000, Phys. Rev. Lett., 84, 2618

Liu, X.-D., Osher, S. \& Chan, T. 1994, Journal of Computational Physics, 115, 200.

Mallat, S.G. 1989a, IEEE Trans, on PAMI, 11, 674

Mallat, S.G. 1989b, Trans. Am. Math. Soc. 315, 69

Matarrese, S. \& Mohayaee, R, MNRAS, 329, 37.

Meyer, Y. 1992, Wavelets and Operators, (Cambridge Press, New York).

Nusser, A. 2000, MNRAS, 317, 902

Olive, K.A., Steigman, G. \& Walker, T.P. 1991, ApJ, 380, L1

Omang, M., Borve, S. \& Trulsen, J. 2003, Computational Fluid Dynamics Journal, 12(2), 32,

Pando, J., Feng, L.L., Jamkhedkar, P., Zheng, W., Kirkman, D., Tytler, D. \& Fang, L.Z., 2002, ApJ, 574, 575

Pando, J., Feng, L.L. \& Fang, L.Z., 2004, ApJ, 154, 475 
Polyakov, A.M. Phys. Rev. E52, 6183, 1995

Ponman, T., Cannon, D., \& Navarro, J. 1999, Nature, 397, 135

Sand, D., Treu, T., Smith, G. and Ellis, R. 2003, ApJ, 604, 88.

Shu, C.-W. 1998, Lecture Notes in Mathematics (Springer), 1697, 325.

Shu, C.-W. 1999, Lecture Notes in Computational Science and Engineering, 9, 439.

Shu, C.-W. 2003 International Journal of Computational Fluid Dynamics, 17, 107-118.

Vergassola, M., Dubrulle, B., Frisch, U. \& Noullez, A. 1994, A\&A 289,325

Vicsek, T. 1992, Fractal Growth Phenomena, (World Scientific, 1992)

Yakhot, V. 1998, Phys. Rev. A57, 1737

Zhan, H., Fang, L.Z., \& Burstein, D. 2003, ApJ, $592, \mathrm{~L} 1$

Zhang, Y.-T., Shi, J., Shu, C.-W. \& Zhou, Y. 2003, Physical Review E, 68, 046709.

This 2-column preprint was prepared with the AAS LATEX macros v5.2. 
Fig. 1. $-v$ vs. $v_{d m}$ at each grid point of samples at redshifts $6,4,2,1,0.5$ and 0 . The two solid lines are, respectively, $v=v_{d m}$, and $v=v_{d m} / 4$.

Fig. 2. $-v$ and $v_{d m}$ at $z=0$ for different density ranges: (1) (1) $\rho_{d m}<1$ (top), (2) $1<\rho_{d m}<5$ (middle), and (3) $\rho_{d m}>5$ (bottom).

Fig. 3. $-v$ and $v_{d m}$ for sample $z=0$. The velocity field is smoothed on scales $d=33.3 / 2^{j} \mathrm{~h}^{-1} \mathrm{Mpc}$, and $j=2,4,6,8$.

Fig. 4. $-v$ and $v_{d m}$ for samples at $z=6,4,2$, $1,0.5,0$, and smoothed on scale $8 \mathrm{~h}^{-1} \mathrm{Mpc}$. The solid line is $v=s v_{d m}$. The $v-v_{d m}$ relation is fitted with line $v=s v_{d m}$ (dashed line). The parameter $s$ is shown in each panel.

Fig. 5.- The relation of $v$ vs. $\rho_{d m}$ (top left); $v$ vs. $\rho_{i g m}$ (top right); $v_{d m}$ vs. $\rho_{d m}$ (bottom left), and $v_{d m}$ vs. $\rho_{\text {igm }}$ (bottom right) at each grid point of the samples at redshift $z=0$. The envelop of the $v-\rho_{d m}$ distribution is given by $v=\left[\log \left(\rho_{d m} / 0.001\right)\right]^{b}$, where $b=2.9$ (dotted); 3.0 (dashed) and 3.1 (solid).

Fig. 6. - The relation of $v$ vs. temperature $T$ (top) and $v_{d m}$ vs. temperature $T$ (bottom) at each grid point of the samples at redshift $z=0$. The envelop of $v$ - $T$ distribution is given by $v=$ $[g \log (T / 100)]^{b}$, where $b=2.5 ; g=2.6$ (solid), 3.0 (dashed) and 3.5 (solid).

Fig. 7.- The PDF of velocity difference $\Delta v_{j, l}$ for acceleration events (solid) and deceleration events (dotted). The samples are at $z=0$ with smoothing scale $d=33.3 / 2^{j} \mathrm{~h}^{-1}$ and $j=3,4,5$ and 6 .

Fig. 8. - The PDF of velocity difference $\Delta v_{j, l}$ for acceleration events (solid) and deceleration events (dotted). The samples are at redshifts $z=4,2,1$ and 0 , with smooth scale is $j=4$.

Fig. 9.- The PDF of velocity difference $\left|\Delta v_{j, l}\right|$ of deceleration cases, i.e. $\Delta v_{j, l}\left(v_{j, l} /\left|v_{j, l}\right|\right)_{d m}<0$ The samples is for $z=0$. The scales $r$ in the velocity difference $\Delta v_{r}=v(x+r)-v(x)$ are taken to be $j=2,3,4,56$ and 7 .

Fig. 10. - The PDF of velocity difference $\left|\Delta v_{j, l}\right|$ of acceleration cases, i.e. $\Delta v_{j, l}\left(v_{j, l} /\left|v_{j, l}\right|\right)_{d m}>0$ The samples is for $z=0$. The scales $r$ in the velocity difference $\Delta v_{r}=v(x+r)-v(x)$ are taken to be $j=2,3,4,56$ and 7 . 
This figure "Fig01.jpg" is available in "jpg" format from: http://arxiv.org/ps/astro-ph/0502175v2 
This figure "Fig02.jpg" is available in "jpg" format from: http://arxiv.org/ps/astro-ph/0502175v2 
This figure "Fig03.jpg" is available in "jpg" format from: http://arxiv.org/ps/astro-ph/0502175v2 
This figure "Fig04.jpg" is available in "jpg" format from: http://arxiv.org/ps/astro-ph/0502175v2 
This figure "Fig05.jpg" is available in "jpg" format from: http://arxiv.org/ps/astro-ph/0502175v2 
This figure "Fig06.jpg" is available in "jpg" format from: http://arxiv.org/ps/astro-ph/0502175v2 
This figure "Fig07.jpg" is available in "jpg" format from: http://arxiv.org/ps/astro-ph/0502175v2 
This figure "Fig08.jpg" is available in "jpg" format from: http://arxiv.org/ps/astro-ph/0502175v2 
This figure "Fig09.jpg" is available in "jpg" format from: http://arxiv.org/ps/astro-ph/0502175v2 
This figure "Fig10.jpg" is available in "jpg" format from: http://arxiv.org/ps/astro-ph/0502175v2 